\title{
Editorial
}

\section{Nano-science and dentistry}

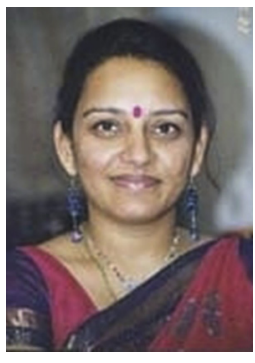

Nanoscience is defined as science dealing with basis of phenomenon of difference in properties of materials and their manipulation at atomic, molecular and macromolecular scales. The scale of being categorized as nano is between 1 to $100 \mathrm{~nm}$. It is an interdisciplinary science both horizontal and vertical in applications.

Nanodentistry is such a new amalgamation of dentistry with nanoscience. It is an emerging field with the potential to generate technologically advanced clinical tools and devices for oral healthcare. Dental implants can be possible in just a single sitting with ultimate correctness using nanodentistry. Diagnoses will be done using nano devices, with solution possible in the shortest span of time. Examples of dental materials include biodegradable nanofibres, nanocrystalline silver particles, nanofillers impregnated impression materials, nanosolutions as bonding agents, nanocomposites. Similarly, for diagnosis purpose as in case of oral cancer Nano Electromechanical Systems (NEMS) are available.

Nanodentistry also refers to robotic dentistry utilizing nanorobots called as 'Dentirobots', 1-10 micron in size and crawling at speed of 1-10 microns/sec. They will help to destroy harmful bacteria on daily basis decreasing tooth decay, cavities and gum problems. Tooth repair, orthodontics, sensitivity gum repair, cosmetic procedures, tooth renaturalization etc. will become possible with this technology. Advantages include their lesser cost, auto-deactivations, if swallowed and programmablility with strict occlusal avoidance protocols.
But there are certain limitations. Technological changes that come along Nanodentistry may demand a degree in nanorobotics. Dental assistant will require know-how in handling nanomaterials and nanorobotics. Though making smaller machine tools, microscopic instruments and manufacturing tools such as nano machines, nano robots, and nano devices may be a lucrative business in future, but the appropriate governmental support is a necessity. The Government of India, in May 2007, has approved the launch of a Mission on Nano Science and Technology (Nano Mission) with an allocation of Rs. 1000 crore for 5 years. At the international level, such support is necessary. Then educational and research framework is required to be laid down and appropriate incentives should be given. Finally, national dental councils are required to play an active role in promoting the use of clinically relevant technologies and offering support to innovative students and practitioners.

Divya Mehrotra

Editor in Chief, Journal of Oral Biology \& Craniofacial Research Professor, Department of Oral \& Maxillofacial Surgery, King George's Medical University, Lucknow, Uttar Pradesh, India E-mail address: divyamehrotra@hotmail.com

http://dx.doi.org/10.1016/j.jobcr.2015.02.005 2212-4268/Copyright @ 2015, Craniofacial Research Foundation. All rights reserved. 